\title{
Parietal Epidural Dermoid Mimicking a Scalp Abscess in an Infant: Case Report
}

\author{
Roka YB ${ }^{1}$, Bajracharya $A^{2}$, Shrestha $K^{3}$, Khaniya $S^{4}$ \\ ${ }^{1}$ Dr. Yam B Roka. M.S, M.Ch (Neurosurgery), FNNI (Skull Base Surgery), Associate Professor, ${ }^{2}$ Dr. A Bajracharya, \\ Resident in Surgery, ${ }^{3}$ Dr. K Shrestha Resident in Surgery, ${ }^{4}$ Dr. S Khaniya, MS, Assistant Professor. All from the \\ Neurosurgical Unit, Department of Surgery. B. P. Koirala Institute of Health Sciences, Dharan, Nepal.
}

Address for Correspondence: Dr. Yam B Roka, E-mail: dryamroka@yahoo.com

\begin{abstract}
Scalp masses that involve the scalp or the cranium especially over the parietal bone are uncommon presentation in infants. We report a case of an 8-week old female child who presented with a left parietal scalp mass which had been progressively increasing since birth. CT scan revealed the mass extending into the cranium with underlying bone defect over the parietal area, with medial extension till the sagittal suture. Intraoperatively there was presence of thick white infected flaky material with small tufts of hair which was removed completely till the bone edges and from the epidural space. There was no recurrence postoperatively and the baby was discharged after a week of antibiotics. This case provides an example that simple looking scalp masses in infants and children should be managed with caution to prevent further morbidity.
\end{abstract}

Key words: Dermoid, Scalp abscess

\section{Introduction}

Darietal area of the scalp is an uncommon location 1 for the dermoid cyst which is usually occur at the angle of the eye ${ }^{1}$. Most of the dermoid reported in literature are in young children and adults ${ }^{2,3,4}$. Scalp masses that involve the scalp or the cranium are uncommon presentation in infants. Apart from the common presentation of lump, they may present with discharging sinus, fistula, malignant transformation or abscess-as in this case report ${ }^{3}$. Improper investigations can lead to misdiagnosis of these lumps with resulting abscess formation as exemplified by our case report.

\section{Case Report}

An 8-week-old female child presented with a left parietal scalp mass which had been progressively increasing since birth. Due to the location of the cyst the mother had difficulty in keeping her baby supine so that the head had taken an elliptical antero-posterior shape.
The baby had undergone treatment elsewhere where fine needle aspiration of the contents was done which had revealed thick white aspirate. Examination revealed a soft cystic mass over the left parietal area near the vertex with ill defined margins, cough impulse was negative and there were no other congenital abnormalities. CT scan revealed the scalp mass extending into the cranium with underlying bone defect over the parietal area with medial extension to the sagittal suture (Fig: 1). The mass although having an intracranial extension was located epidurally. Intraoperatively there was presence of thick white infected material with small tufts of hair which was removed completely till the bone edges and from the epidural space. The culture and Gram stain showed Staphylococcus aureus which was sensitive to cloxacillin. Histopathology was consistent with dermoid. There was no recurrence postoperatively and the baby was discharged after a short course of antibiotics. 


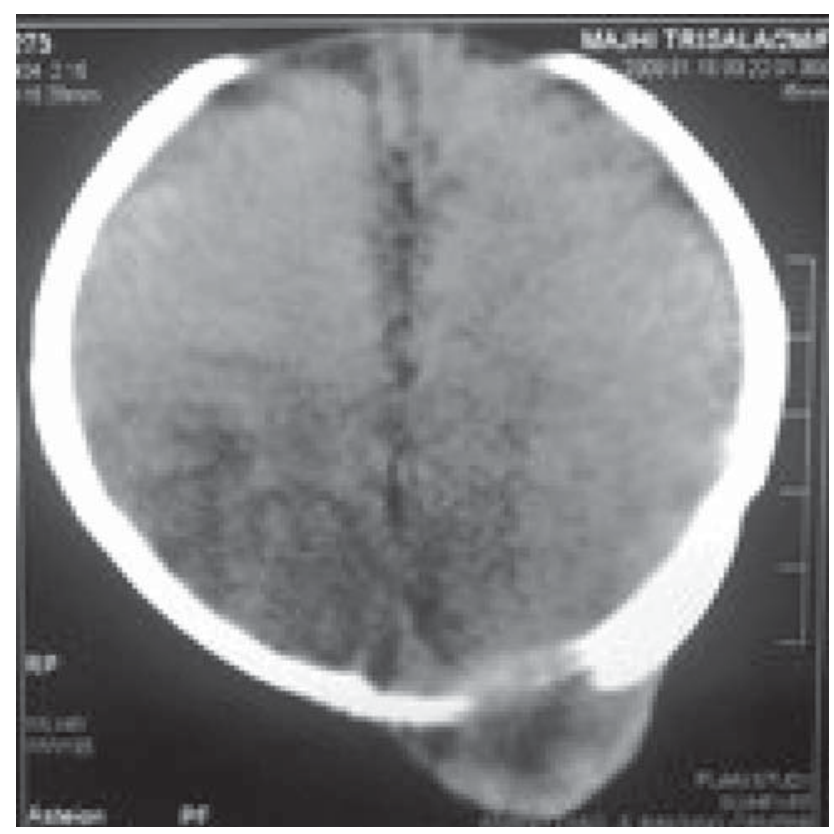

Fig 1: CT scan showing left parietal defect with the dermoid extending to the epidural space.

\section{Discussion}

Parietal area of the scalp is an uncommon location for the dermoid cyst which is usually occur at the angle of the eye ${ }^{1}$. Most of the dermoid reported in literature are in young children and adults ${ }^{2,3,4}$. The average age at presentation was 35 years for patients with epidermoids and 15 years for those with dermoids in a series by Gromley et al. ${ }^{2}$ Subgaleal dermoid cyst is rare in childhood and exceptionally occurs in adults with only six cases mentioned in the literature ${ }^{5}$. Very rarely the dermoid can have an intracranial extention ${ }^{2.5 .6}$. Of the varied differential for a scalp lump in infants is infantile myofibromatosis, a disorder of infancy and early childhood, typically presenting as a solitary lesion or multiple widespread nodular tumors localized to skin, subcutaneous tissue, muscle, bone, viscera, or central nervous system ${ }^{7}$. Discharging sinus or a fistula is another rare presentation of scalp dermoids. In the case reported by Parag et al, the fronto-temporal dermoid was associated with both discharging sinus and also presence of intracranial extention ${ }^{8}$. A single case of sublingual dermoid with malignant transformation has also been reported in the literature ${ }^{8}$. Posterior fossa dermoid with sinus and meningitis in a toddlerand 2-year old has also been reported ${ }^{9,10}$.

Surgery is the preferred method of treatment and since they behave in a benign manner total excision is curative. Those lesions with intracranial extension warrant further investigation with MRI to delineate the tumor and surrounding structures and if near to neurovascular structures then complete resection should not be the goal to avoid damage to these latter structures. This is in contrast to the conclusion by Fornari et al who treated thirty patients with scalp epidermoid and dermoids with only two recurrences. They concluded by saying that even when these tumors are connected to vital neurovascular structures, total excision seems advisable in the first two decades of life $^{11}$. Newer minimally invasive and cosmetically acceptable endoscopic technique for removal of lesions over the frontal area has also been described ${ }^{12}$.

Although uncommon in infants dermoid cyst must be kept as a possibility in all scalp masses and preoperative imaging done to prevent further morbidity. Especially in this regards intracranial extension needs to be ruled out to prevent damage to brain or development of intracerebral abscess/meningitis. This has major implications in developing countries where treatment is mainly based on clinical examination and where radiological investigations may not be widely available.

\section{Acknowledgement: None}

Funding: None

\section{Conflict of Interest: None}

\section{References}

1. Schwartz R A, Ruszczak Z. Dermoid Cyst; eMedicine; Jun 2007. Accessed Jan 2009. http:// emedicine.medscape.com/article/1112963overview. 
2. Gormley WB, Tomecek FJ, Qureshi N, Malik GM. Craniocerebral epidermoid and dermoid tumours: a review of 32 cases. Acta Neurochir. 1994; 128(14):115-21.

3. Adouani A, Chennoufi M, Hammoud M, et al. Malignant transformation of a dermoïd cyst of the scalp. A case report. Tunis Med. 2004 Oct; 82(10):972-5.

4. Cummings TJ, George TM, Fuchs HE, McLendon RE. The pathology of extracranial scalp and skull masses in young children. Clin Neuropathol. 2004 Jan-Feb; 23(1):34-43.

5. Hubault-Marcade P, Hepner-Lavergne D, Pannier M. Dermoid cyst of the scalp. Apropos of a case. Ann Chir Plast Esthet. 1991; 36(5):452-6.

6. Crawford R. Dermoid cyst of the scalp: intracranial extension. J Pediatr Surg. 1990 Mar; 25(3):294-5.

7. Huang CJ, Lin KL, Jung SM, Wu CT, Wang HS. Infantile myofibromatosis presenting with scalp dermoid cyst.Pediatr Neurol. 2005 Oct; 33(4):2969.
8. Parag P, Prakash PJ, Zachariah N. Temporal dermoid--an unusual presentation. Pediatr Surg Int. 2001; 17(1):77-9.

9. Douvoyiannis M, Goldman DL, Abbott IR 3rd, Litman N. Posterior fossa dermoid cyst with sinus tract and meningitis in a toddler. Douvoyiannis M, Goldman DL, Abbott IR 3rd, Litman N.Pediatr Neurol. 2008 Jul; 39(1):63-6.

10. Cai CQ, Zhang QJ, Hu XL, Wang CX. Dermoid cyst of the posterior fossa associated with congenital dermal sinus in a child. World J Pediatr. 2008 Feb; 4(1):66-9.

11. Fornari M, Solero CL, et al. Surgical treatment of intracranial dermoid and epidermoid cysts in children. Childs Nerv Syst. 1990 Mar; 6(2):66-70.

12. Dutta S, Lorenz HP, Albanese CT. Endoscopic excision of benign forehead masses: a novel approach for pediatric general surgeons.J Pediatr Surg. 2006 Nov; 41(11):1874-8. 